C. Beckermann

Associate Professor.

T. F. Smith

Professor.

Department of Mechanical Engineering, The University of lowa, lowa City, lowa 52242

\section{B. Pospichal}

Air Transport Systems, Rockwell International Corporation, Cedar Rapids, lowa 52498

\section{Use of a Two-Dimensional} Simulation Model in the Thermal Analysis of a Multi-Board Electronic Module

A study is reported of heat transfer and air flow in an electronic module consisting of an array of narrowly spaced vertical circuit boards with highly-protruding components contained in a naturally vented chassis. A two-dimensional simulation model is developed that accounts for heat transfer by conduction, convection, and radiation, and sensitivity studies are performed. Experiments are conducted using a specially constructed test module. Comparsions with the experiments reveal the need to calibrate the model by selecting an effective component height that represents the drag properties of the actual three-dimensional component geometry. The need to account in the model for heat losses in the depth direction is also discussed. The importance of accurate thermophysical properties and of multi-dimensional radiation is shown. Good agreement with measured velocities and local board temperatures is obtained over a wide range of power levels, and it is concluded that the calibrated model is capable of representing the thermal behavior of the present module.

\section{Introduction}

The trend toward denser packaging and higher heat dissipation has resulted in renewed attention to the analysis of the thermal performance of electronic equipment. Natural convection cooling of arrays of printed circuit boards continues to be the thermal management technique of choice for many electronic applications. While a model of the combined conduction, natural convection, and radiation heat transfer in a sealed electronic chassis was reported in a previous study (Smith et al., 1991), this paper is concerned with the modeling of an open cabinet containing an array of vertically oriented printed circuit boards (PCBs) on which discrete heat generating components are mounted. In this configuration, flow is induced into the channels between the PCBs by the so-called "chimney" effect.

There have been numerous studies on electronic cooling by natural convection between vertical circuit cards, and a recent review is available (Peterson and Ortega, 1990). A combined numerical and experimental investigation was reported by Aung et al. (1972) who developed a design methodology for estimating card temperatures and choosing the optimum card spacing. The effects of real components protruding from the PCB, staggered cards, and baffles were also discussed. Birnbreier (1981) carried out experimental work using fully assembled PCBs, which were mounted in modules. It was found that a model based on a smooth channel with uniform wall heat

Contributed by the Electrical and Electronic Packaging Division for publication in the Journal of Electronic Packaging. Manuscript received by the EEPD June 1, 1993; revised manuscript received January 4, 1994. Associate Technical Editor: W. Z. Black. flux cannot be used to calculate PCB temperatures and that radiation heat transfer strongly reduces the maximum temperature of the PCBs at the highest level. Coyne (1984) later presented a simplified analysis of the radiative and conductive heat transfer between PCBs and reported good agreement with the experimental data of Birnbreier (1981). In a series of studies, Ortega and Moffat $(1985,1986)$ and Moffat and Ortega (1986) investigated in detail the convective mechanisms in a vertical channel with an array of protruding, heat generating elements at discrete sites on one wall. They proposed a new thermal design method that takes into account the effects of the discretized heat dissipation and hydrodynamic drag by the elements. However, the drag properties must be obtained from a measurement of the pressure drop at a known (forced) flow.

An important limiting case of the configuration considered here is a vertical channel formed by smooth parallel plates. A fairly complete tabulation of the many studies performed on this simplified geometry can be found in Peterson and Ortega (1990). Bar-Cohen (1985) demonstrated how analytical, smooth-plate relations can be used in the development of initial designs to estimate maximum component temperatures. However, the procedure becomes increasingly inaccurate as the ratio of the component height to the channel width increases and the flow "arteries" formed by the spaces between components grow in importance. Again, it was noted that for high ratios, it is necessary to quantify the flow-resistance characteristics of the PCB surface. Another approach is exemplified by the recent work of Hawkins and Nelson (1992), who numerically solved the fully elliptic flow and energy equations to account for inlet and exit effects in short channels and the possible occurrence of flow recirculation, and included radiation heat 
transfer in their analysis. The calculations revealed that the fully-developed-flow assumption and the neglect of either the buoyancy or the radiant effects cannot be justified in electronic cooling analyses. The study was limited to a single smooth channel with a uniform heat flux on one wall and an insulated opposite wall.

While analytical methods are available for the initial thermal design of naturally cooled PCB modules, the greater flexibility and potential for more detailed information on local heat transfer paths and temperatures offered by numerical simulation models make them useful, in more advanced design stages. Nonetheless, no model has been identified that solves the coupled flow and energy equations (as in Smith et al., 1991; Hawkins and Nelson, 1992) and can deal with realistic electronic assemblies having a three-dimensional arrangement of discrete components on multiple PCBs. In fact, the fine grid required in such cases would exceed the capabilities of even modern computer workstations. Therefore, the present study was undertaken to explore the utility of a two-dimensional simulation model. Experimental data from a representative, though simple, multi-board module with discrete heat generating components are used to establish the feasibility and steps necessary to represent accurately the actual three-dimensional flow and heat transfer in a two-dimensional model. Attention is focused on a relatively high component height to channel width ratio of 0.61 . The study also addresses other modeling issues that are equally important in future three-dimensional models, such as the effects of variable air properties, the choice of effective thermal conductivities for certain heterogeneous materials, and the need to account for multi-dimensional radiation. The latter item is of particular interest, as most previous studies (Peterson and Ortega, 1990) either neglect radiation or use a much more simple one-dimensional model.

\section{Model}

2.1 System. The two-dimensional system selected for analysis is shown in Fig. 1, where the various design parameters are defined in Table 1 . Subscripts of $i$ and $j$ on several of the parameters refer to the $x$-and $y$-directions, respectively, and are used to infer that these quantities do not need to be uniform for the entire module. The module consists of eight printed circuit boards (PCBs) on each of which are mounted eight heat generating components in the vertical direction. There are uniform separations between the PCBs and the components. All components have the same size, thermophysical properties, and heat generation rates. The PCBs are conductively isolated

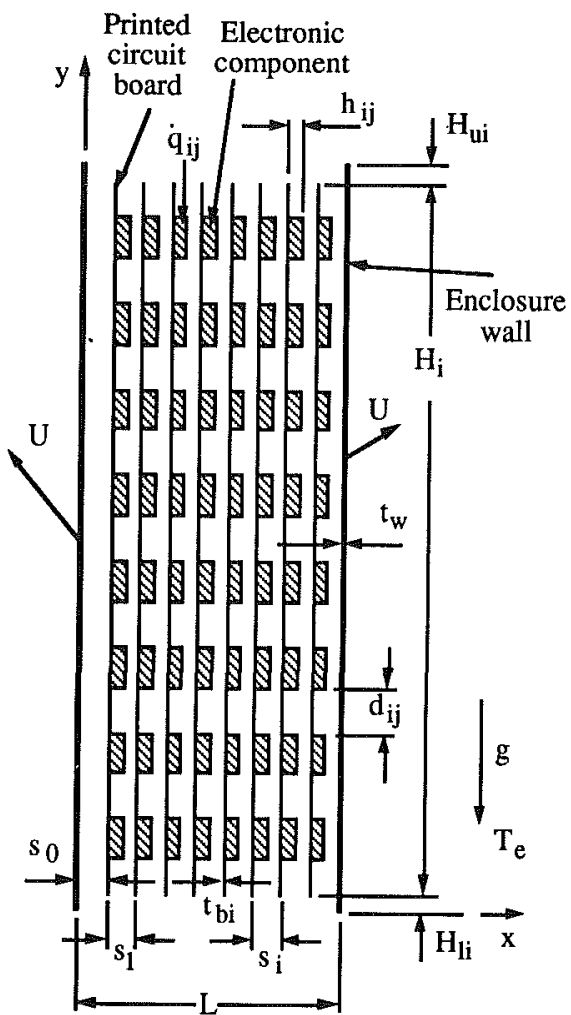

Fig. 1 Schematic of the two-dimensional system used in the numerical model

from the module walls and each other. The left and right sides of the module are constructed of impermeable plates.

The ambient air is at a temperature $T_{a}$ and atmospheric pressure. The air flow through the module is assumed to be laminar and steady, and gravitational acceleration acts parallel to the $y$-direction. Air properties are temperature dependent and are taken from the values cited in Incropera and DeWitt (1990). Temperature independent properties are assumed for all solid materials. For the purpose of evaluating the radiative heat transfer, the air is transparent with a refractive index of unity, and all solid surfaces are diffuse, gray, and opaque.

The overall heat transfer coefficient between the walls and the external surroundings is assumed to be constant and uniform. The external surroundings are large and at a temperature $T_{e}$. Radiant exchange takes place between internal surfaces of

\section{Nomenclature}

$$
\begin{aligned}
A_{b}, A_{f}= & \text { back and front areas, } \mathrm{m}^{2} \\
c_{p}= & \text { constant pressure specific } \\
& \text { heat, } \mathrm{kJ} / \mathrm{kg}-\mathrm{K} \\
d= & \text { separation distance and } \\
& \text { component length, } \mathrm{m} \\
g= & \text { gravitational acceleration, } \\
& \mathrm{m} / \mathrm{s}^{2} \\
h= & \text { component height, } \mathrm{m} \\
H= & \text { length of printed circuit } \\
& \text { board, } \mathrm{m} \\
H_{1}, H_{u}= & \text { board recess from bottom } \\
& \text { or top of enclosure, } \mathrm{m} \\
I= & \text { radiant intensity, } \mathrm{W} / \mathrm{m}^{2}-\mathrm{sr} \\
k= & \text { thermal conductivity, } \mathrm{W} / \mathrm{m}- \\
& \text { K } \\
L= & \text { width of system, } \mathrm{m} \\
P= & \text { pressure, } \mathrm{N} / \mathrm{m}^{2} \\
q= & \text { heat transfer rate, } \mathrm{W}
\end{aligned}
$$

$$
\begin{aligned}
\kappa= & \text { absorption coefficient, } \mathrm{m}^{-1} \\
\mu= & \text { dynamic viscosity, } \mathrm{kg} / \mathrm{s}-\mathrm{m} \\
\rho= & \text { density, } \mathrm{kg} / \mathrm{m}^{3}, \text { and reflec- } \\
& \text { tance } \\
\phi= & \text { polar angle, deg } \\
\omega= & \text { solid angle, } \mathrm{sr}
\end{aligned}
$$

Subscripts

$$
\begin{aligned}
a & =\text { ambient } \\
b & =\text { blackbody } \\
c & =\text { component } \\
e & =\text { surroundings } \\
e x & =\text { exit } \\
f b & =\text { front/back } \\
i n & =\text { inlet } \\
p & =\text { printed circuit board } \\
s & =\text { surface }
\end{aligned}
$$$$
w=\text { chassis wall }
$$ 
Table 1 Notation for Fig. 1 and parameters for base case

\begin{tabular}{llr}
\hline$d_{i j}$ & Separation distance and component length \\
& $j$-odd: 1 to 17 (separation distance) & $19.87 \mathrm{~mm}$ \\
& $j$ - even: 2 to 16 (component length) & $9.40 \mathrm{~mm}$ \\
$h_{i j}$ & Physical height of component & $3.89 \mathrm{~mm}$ \\
$h_{c}$ & Height of component in model & $1.40 \mathrm{~mm}$ \\
$H_{l i}$ & Board recess from bottom of enclosure & $6.35 \mathrm{~mm}$ \\
$H_{u i}$ & Board recess from top of enclosure & $6.35 \mathrm{~mm}$ \\
$g$ & Gravitational acceleration & $9.81 \mathrm{~m} / \mathrm{s}^{2}$ \\
$k_{b i}$ & Thermal conductivity of printed circuit board & \\
& $x$-direction & $10.38 \mathrm{~W} / \mathrm{m}-\mathrm{K}$ \\
& $y$-direction & $10.38 \mathrm{~W} / \mathrm{m}-\mathrm{K}$ \\
$k_{c i j}$ & Thermal conductivity of component & $86.52 \mathrm{~W} / \mathrm{m}-\mathrm{K}$ \\
$k_{w}$ & Thermal conductivity of chassis walls & $173.03 \mathrm{~W} / \mathrm{m}-\mathrm{K}$ \\
$\dot{q}_{i j}$ & Heat generation rate & $9.92 \times 10^{5} \mathrm{~W} / \mathrm{m}$ \\
$s_{i}$ & Printed circuit board spacing & $6.35 \mathrm{~mm}$ \\
& $i=0$ & $6.35 \mathrm{~mm}$ \\
& $i=1$ to 8 & $2.21 \mathrm{~mm}$ \\
$t_{b i}$ & Thickness of printed circuit board & $1.21 \mathrm{~mm}$ \\
$t_{w}$ & Thickness of enclosure wall & $24{ }^{\circ} \mathrm{C}$ \\
$T_{a}$ & Temperature of inlet air & $24{ }^{\circ} \mathrm{C}$ \\
$T_{e}$ & Temperature of surroundings & 0.8 \\
$U_{i}$ & Overall heat transfer coefficients & 0.8 \\
$\epsilon_{c i}$ & Emittance of components & 0.25 \\
$\epsilon_{p i}$ & Emittance of printed circuit board & $10.25 \mathrm{~W} / \mathrm{m}$ \\
$\epsilon_{w}$ & Emittance of interior chassis wall & \\
\hline
\end{tabular}

the module and the external surroundings through the openings at the top and bottom and is evaluated using $T_{e}$.

2.2 Governing Equations. In the same manner as in Smith et al. (1991), a single solution domain procedure is employed, in which one set of conservation equations is solved over the two-dimensional region shown in Fig. 1, and the thermophysical properties depend on the material present at a particular location. The conservation equations can be written as Continuity:

$$
\frac{\partial}{\partial x}(\rho u)+\frac{\partial}{\partial y}(\rho v)=0
$$

$x$-momentum:

$$
\rho u \frac{\partial u}{\partial x}+\rho v \frac{\partial u}{\partial y}=\frac{\partial}{\partial x}\left(\mu \frac{\partial u}{\partial y}\right)+\frac{\partial}{\partial y}\left(\mu \frac{\partial u}{\partial y}\right)-\frac{\partial P}{\partial x}
$$

$y$-momentum:

$$
\begin{aligned}
\rho u \frac{\partial v}{\partial x}+\rho v \frac{\partial v}{\partial y}=\frac{\partial}{\partial x}\left(\mu \frac{\partial v}{\partial x}\right)+\frac{\partial}{\partial y}\left(\mu \frac{\partial v}{\partial y}\right)-\frac{\partial P}{\partial y} & \\
& +\rho g \beta\left(T-T_{a}\right)
\end{aligned}
$$

Energy:

$$
\begin{aligned}
\rho u c_{p} \frac{\partial T}{\partial x}+\rho v c_{p} \frac{\partial T}{\partial y}=\frac{\partial}{\partial x}(k & \left.\frac{\partial T}{\partial x}\right) \\
& +\frac{\partial}{\partial y}\left(k \frac{\partial T}{\partial y}\right)-\nabla \cdot q_{r}+\dot{q}-\dot{q}_{f b}
\end{aligned}
$$

where the pressure $P$ is defined as the pressure defect relative to the ambient pressure. In solid materials, the viscosity is set to a very large value in order to force the velocities to vanish. This procedure for handling conjugate heat transfer problems is discussed in more detail in Patankar (1980). The fourth and fifth term on the right-hand side of Eq. (4) are the volumetric heat generation rate of the components and the front/back heat loss rate, respectively. The third term on the right-hand side of Eq. (4) is the divergence of the radiative heat flux. Because the air is assumed to be radiatively transparent and the solid materials to be opaque, the radiative flux is nonzero only at air/solid interfaces.

The boundary conditions for the flow are given by a specified pressure defect at the inlet and exit, that is

$$
P_{i n}=-\frac{1}{2} \rho \bar{v}^{2} ; \quad P_{e x}=0
$$

where $\bar{v}$ is the mean normal velocity at the inlet. The negative inlet pressure defect is caused by the acceleration of the air from rest to the inlet plane. In addition, the normal velocity gradients at the inlet and exit are equal to zero. The thermal boundary conditions are given by a uniform specified inlet temperature and a vanishing normal temperature gradient at the exit. At the solid walls of the module, the heat loss is given as the product of the overall heat transfer coefficient, $U$, and the local temperature difference between the wall and the surroundings.

2.3 Radiation Fluxes. Sánchez and Smith (1992) developed the discrete-ordinates method for surfaces separated between transparent media. The basic equations used in the method are described in this section with the solution procedure discussed in Section 2.4.

The two-dimensional radiative transport equation for the intensity for an absorbing and emitting medium within the solution domain is

$$
\zeta \frac{\partial I}{\partial x}+\eta \frac{\partial I}{\partial y}=-\kappa I+\kappa I_{b}
$$

where $I=I(x, y ; \zeta, \eta), \zeta$ and $\eta$ are direction cosines, $\kappa$ is the absorption coefficient of the medium, and $I_{b}$ is the blackbody intensity. Opaque and transparent media are presented by large and zero values of the absorption coefficient, respectively. For a large value of the absorption coefficient, the intensity equals the blackbody intensity, and for an absorption coefficient of zero, the intensity remains unchanged. At an opaque surface, the intensity leaving is composed of the emitted intensity and the reflected irradiation and is written as

$I\left(x_{s}, y_{s} ; \zeta, \eta\right)=\epsilon_{s} I_{b}\left(x_{a}, y_{a}\right)$

$$
+\frac{\rho_{s}}{\pi} \int_{2 \pi} I^{\prime}\left(x_{s}, y_{s} ; \zeta^{\prime}, \eta^{\prime}\right) \cos \phi^{\prime} d \omega^{\prime}
$$

where subscript " $s$ " denotes a surface, $\epsilon$ is the surface emittance, and $\rho_{s}$ is the surface reflectance $\left(=1-\epsilon_{s}\right)$. The prime denotes an incoming quantity, $\phi$ is the polar angle between the surface normal and the intensity, and $\omega$ is the solid angle. The integration in Eq. (10) is performed over the hemisphere above the surface.

The net radiant energy leaving an opaque surface is

$$
\begin{aligned}
& q_{r}\left(x_{s}, y_{s}\right)=\int_{2 \pi} I\left(x_{s}, y_{s} ; \zeta, \eta\right) \cos \phi d \omega \\
&-\int_{2 \pi} I^{\prime}\left(x_{s}, y_{s} ; \zeta^{\prime}, \eta^{\prime}\right) \cos \phi^{\prime} d \omega^{\prime}
\end{aligned}
$$




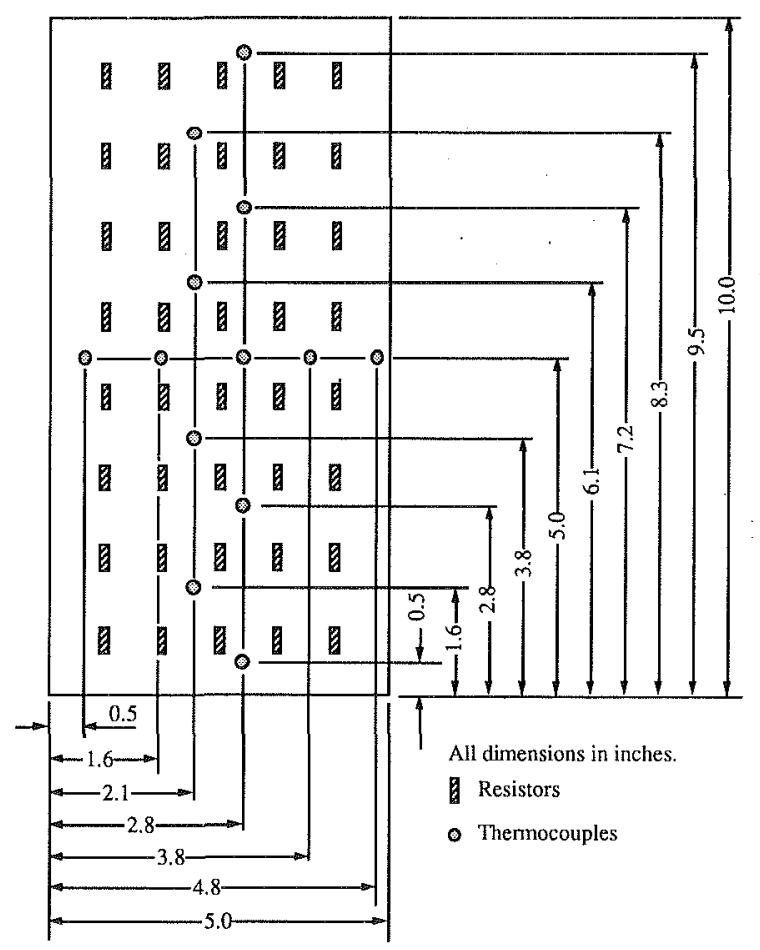

Fig. 2 Schematic of the test board showing resistor and thermocouple locations

which represents the difference between the leaving and arriving radiant energy.

2.4 Numerical Solution Procedures. The finite volume method of Patankar (1980) is used to solve the conservation equations. The combined advective and diffusive fluxes are discretized using the power-law scheme and the pressure/velocity coupling is affected through the SIMPLER algorithm. The harmonic mean formulation adopted for the interface diffusion coefficients between two control volumes yields physically realistic results for abrupt changes in these coefficients (viscosity, thermal conductivity) without requiring an excessively fine grid in the neighborhood of the air/solid interfaces. Special procedures are required to incorporate the surface radiative heat fluxes into the method (Beckermann and Smith, 1993). The calculation of the radiative heat fluxes is based on the discrete-ordinates method specially developed for application to radiant exchange between surfaces separated by a transparent medium (Sánchez and Smith, 1992). The method allows a surface to view all other surfaces in a hemisphere over the surface. An opaque component is simply defined as a region with a high absorption coefficient. The method has the same grid as that used for the flow and heat transfer solver. Convergence of the numerical solution was checked by performing overall mass and energy balances.

The numerical code was validated through extensive comparisons with results for limiting cases available in the literature (Beckermann and Smith, 1991). Good agreement was obtained for pure natural convection and combined natural convection and radiation in a square cavity and in a single vertical channel. At the same time, these tests helped to establish the grid density necessary to obtain accurate solutions for the conditions of the present study. For the base case (discussed later), a grid of $134 \times 94$ control volumes in the $x$ - and $y$-directions was utilized, with 14 control volumes staggered across each of the spaces between the PCBs. Other details can be found in Beckermann and Smith (1992). The calculations were performed on an Apollo DN10000 workstation and typically required approximately one hour of CPU time.

\section{Experiments}

Experiments were performed using a test setup as shown in Fig. 1 (Thornton, 1987). The eight PCBs çonsist of ten layer polymide with two, 2.8 mil ( 2 oz.) copper planes. The PCBs

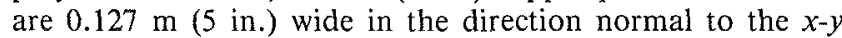
plane. As shown in Fig. 2, each PCB is loaded with forty, one watt carbon resistors in eight columns of five resistors. All dimensions can be found in Table 1 . The assembly is enclosed with $1.524 \mathrm{~mm}(0.153 \mathrm{in}$.) thick aluminum sides. The rear cover is attached directly to the boards simulating a rear interconnect board, while the front cover is spaced $6.35 \mathrm{~mm}(0.25 \mathrm{in}$.) away from the boards.

Figure 2 shows the thermocouple locations for the test board. Each PCB has nine evenly spaced thermocouples in the vertical direction and five across the board. The thermocouples are 30 gauge copper-constantan wire with welded beads that are attached with Armstrong's A2. The load resistors are powered with a DC power supply and the current is measured using DC shunt resistors. All temperature and power measurements are recorded with a Fluke 2240 data logger. The thermocouples were calibrated with an accuracy of $\pm 1.5 \mathrm{~K}$. The mean air velocity through the module was measured by scanning the exit plane with a Thermal Systems Incorporated Model 1650 anemometer. Ambient conditions were monitored throughout the tests. Before the measurements were undertaken, the setup was allowed to reach a steady state for several hours.

\section{Numerical Results}

This section presents representative model results and examines their sensitivity to changes in several input parameters, such as the various thermophysical properties. In addition, the importance of radiation is clarified. The sensitivity studies are useful when the model results are compared to the experimental findings in Section 5.

4.1 Base Case. The attributes of the base case are listed in Table 1. The front/back heat loss rate is set to zero, the air properties are fully temperature dependent, and all heat transfer modes are included. Noteworthy is the assigned height of a component of $h_{c}=1.397 \mathrm{~mm}$, whereas the physical height is $3.886 \mathrm{~mm}$. This reduced height arises from the necessity to average the component (that is, resistor) height over the depth of the PCB in the two-dimensional model (compare Figs. 1 and 2), and results in realistic board temperature levels. A detailed discussion of the component height selection is provided in Section 5.

Streamlines and isotherms computed for the base case are displayed in Fig. 3. The streamlines (Fig. 3(a)) in the left-most space are nearly vertical indicating no recirculations. In the other spaces, the streamlines are more concentrated in the flow passages between a component and a PCB. A more detailed examination of the computed velocities (not shown here) showed a weak recirculation above each component in Fig. 1. The isotherms (Fig. 3(b)) show an increasing overall temperature in the vertical direction. As expected, the highest temperatures are found near the top center of the system, where the temperature is nearly uniform. Near the bottom at a given vertical location, the air temperatures lag the PCB and component temperatures, and the air flowing in the spaces has an approximately parabolic temperature profile. The center four PCBs have nearly identical temperatures. Large temperature gradients in the horizontal direction are observed in the air spaces adjacent to the chassis walls.

Table 2 gives an average outlet velocity and board temperature rise of $0.119 \mathrm{~m} / \mathrm{s}$ and $63.83 \mathrm{~K}$, respectively, for the base case. These results are used below as a basis for comparison with the sensitivity studies. The average outlet velocity is obtained by simple integration of the vertical velocities at the exit of the module. On the other hand, the average board tem- 

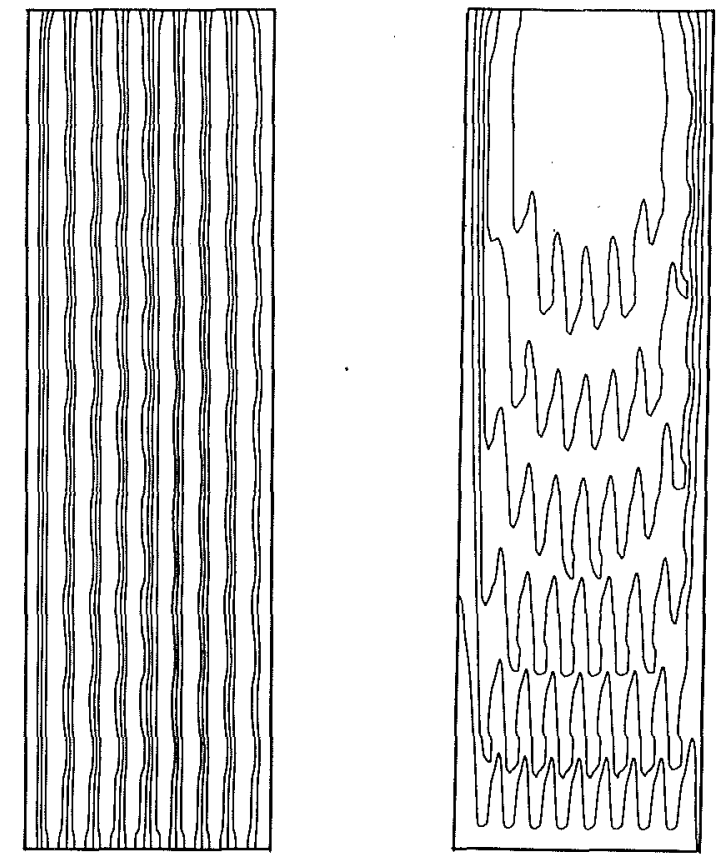

Fig. 3 Streamlines and isotherms for base case. (a) Streamlines (min: - $8.128 \mathrm{~kg} / \mathrm{m}-\mathrm{s} ;$ max: $0.0 \mathrm{~kg} / \mathrm{m} \cdot \mathrm{s} ; 28$ equal increments). (b) Isotherms (min: 297.0 K; max: 398.4 K; increments: $7 \mathrm{~K}$ ).

perature rise is defined as the arithmetic mean of the temperatures of the eight PCBs at the locations of the nine vertically spaced thermocouples on each board (see Fig. 2). The term "rise" implies that the temperatures are relative to (above) the ambient air temperature, $T_{a}$.

4.2 Variable Versus Fixed Air Properties. The effect of neglecting the temperature dependency of the air properties is demonstrated by Case 2 in Table 2 . In Case 2 the thermophysical properties of air are evaluated at a temperature of $330.0 \mathrm{~K}$, which approximately corresponds to the mean temperature of the air in the module for the base case. In comparison to the base case (Case 1), only a slight decrease is observed for the mean outlet velocity. However, the average board temperature rise decreases by 12.7 percent (or $8.3 \mathrm{~K}$ ). In view of the latter finding, temperature dependent air properties must be accounted for. In fact, other choices of the temperature for evaluation of the air properties (such as the "film" or inlet temperatures) will not produce significantly better agreement with Case 1.

4.3 Effect of Thermal Conductivities. Since the PCBs and the components (resistors) are inhomogeneous and consist of multiple materials, their effective thermal conductivities must be estimated. This section examines the sensitivity of the overall model results to changes in these parameters.

The effect of changing the thermal conductivity of the PCBs is illustrated by Cases 3 and 4 in Table 2, where the PCB thermal conductivity listed in Table 1 is altered by multiplicative factors of 10 and 0.1 , respectively. For the higher PCB thermal conductivity (Case 3 ), the mean outlet velocity increases by 5.9 percent, while the average board temperature rise increases by 11 percent (or $7.2 \mathrm{~K}$ ). For the lower $\mathrm{PCB}$ thermal conductivity (Case 4), the mean outlet velocity decreases by 2.5 percent, while the average board temperature rise decreases by 8.1 percent (or $5.3 \mathrm{~K}$ ). Hence, as the thermal conductivity of the PCBs increases, more heat is conducted away from the heat generating components to the PCBs, which become hotter. This causes an increase in the heat transfer to the air, thereby increasing the air velocity due to enhanced buoyancy. Cases 3 and 4 strongly suggest that reasonably ac-
Table 2 Sensitivity findings

\begin{tabular}{clcc}
\hline $\begin{array}{c}\text { Case } \\
\#\end{array}$ & Description & $\bar{v}_{o}$ & $\Delta \bar{T}_{b}$ \\
$\mathrm{~m} / \mathrm{s}$ & $\mathrm{K}$ \\
\hline 1 & Base case & 0.119 & 65.2 \\
2 & Constant air properties & 0.120 & 56.9 \\
3 & PCB thermal conductivity $\times 10$ & 0.126 & 72.4 \\
4 & PCB thermal conductivity $\times 0.1$ & 0.116 & 59.9 \\
5 & Component thermal conductivity $\times 0.1$ & 0.119 & 64.9 \\
6 & Absence of radiation & 0.123 & 69.6 \\
\hline
\end{tabular}

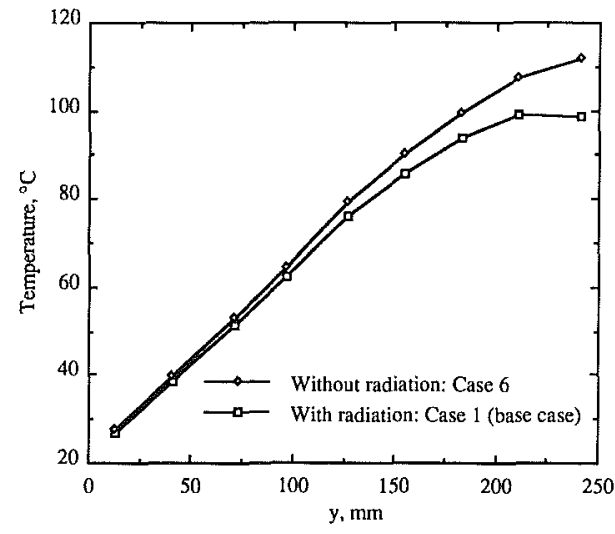

Fig. 4 Local board temperatures of PCB4 Illustrating effect of radiation

curate estimates of the PCB thermal conductivities should be made.

Results for Case 5 in Table 2 show the effect of lowering the component thermal conductivity by a factor of 10 from that given in Table 1. Overall, there is a negligibly small difference between the results for this case and those for the base case. Even the component temperatures (not cited here) decrease, on the average, by only 0.4 percent. Hence, only a rough estimate of the component thermal conductivity is required in the model.

4.4 Effect of Radiation. Significant differences between the results of the base case (Case 1) with radiation and those of Case 6 without radiation are found in Table 2. The results for Case 6 can also be interpreted as illustrating the effects of the emittance, where, for no radiation, the emittance is zero for all surfaces. The average outlet velocity and board temperature rise are 3.4 and 6.7 (or $4.4 \mathrm{~K}$ ) percent higher, respectively, without radiation than with radiation. These findings indicate that it is important to account for radiation in the heat transfer analysis.

Further evidence of the effect of radiation is shown in Fig. 4 , where the local board temperatures at the locations of the nine vertically spaced thermocouples on PCB 4 are plotted for Cases 1 and 6 . Near the bottom of the PCB, the temperatures from both cases are similar due to the small rise of the board temperatures adjacent to the inlet above the temperature of the surroundings, $T_{e}$. However, at the top of the PCB there are large radiation losses to the surroundings, causing the board temperatures for Case 1 to reach a maximum and then decrease. On the other hand, for Case 6 the board temperatures near the top are more than $10 \mathrm{~K}$ higher and do not decrease. In fact, a decrease of the PCB temperatures towards the exit is not possible when radiation is neglected. Furthermore, this decrease could not be predicted with a much more simple onedimensional radiation model that accounts for radiation in the horizontal direction (i.e., between the PCBs) only.

\section{Comparisons With Experiments}

In comparing the experimental results to the predictions of the two-dimensional model, two issues need to be addressed: 
Table 3 Comparison of measured and predicted average board temperature rises and measured outlet velocities for different power levels $\left(h_{c}=1.473 \mathrm{~mm}, \dot{q}_{f b}=0\right)$.

\begin{tabular}{cccc}
\hline & $\dot{q}$ & Experiment & Simulation \\
Case & $10^{5}$ & $\Delta \bar{T}_{b}$ & $\Delta \bar{T}_{b}$ \\
\hline 7 & $\mathrm{~W} / \mathrm{m}^{3}$ & $\mathrm{~K}$ & $\mathrm{~K}$ \\
8 & 9.383 & 67.2 & 67.0 \\
9 & 5.117 & 43.8 & 42.6 \\
10 & 2.560 & 27.4 & 26.6 \\
\hline
\end{tabular}

(i) the discrete nature of the components over the depth of each PCB at a given vertical location (see Fig. 2) and (ii) the heat losses from the front and back walls of the module. These items are discussed separately in the following two subsections.

5.1 Selection of Component Height. From Fig. 2, it can be seen that for each of the eight resistor columns a significant portion of a PCB in the depth direction is not covered by a component. To account for this, it would seem necessary to reduce the component height in the two-dimensional model. A preliminary simulation using the actual height of the component $\left(h_{c}=3.886 \mathrm{~mm}\right)$ yielded an average board temperature rise that was more than twice as large as the measured one (Beckermann and Smith, 1991), mainly because the predicted air flow was too restricted (recall that the board spacing is only $6.35 \mathrm{~mm}$, see Table 1). Several techniques for selecting a more realistic component height for use in the two-dimensional model were examined (Beckermiann and Smith, 1991). Referring to Fig. 2, the component height averaged over the depth of the $\mathrm{PCB}$ at the vertical location of a resistor column is equal to $h_{c}=5(3.886)^{2} / 127.0 \mathrm{~mm}=0.594 \mathrm{~mm}$. The use of this simple geometry-based average gave an average board temperature rise that was about 13 percent too low compared to the experimental value. Although it appears to be possible to select an effective component height that gives an average board temperature rise accurate to within several degrees, such agreement may not be acceptable when using a numerical simulation in more advanced design stages. On the other hand, the development of a general component height selection technique for an arbitrary three-dimensional placement of various components on a PCB is outside the scope of the present study. Therefore, for the purpose of the following comparisons, a calibration procedure was devised, based on a single experiment and the component height as the adjustable parameter.

Comparisons were carried out for four experiments with different power levels. These experiments are denoted as Cases 7 to 10 , and Table 3 lists their respective volumetric heat generation rates (for a component height of $h_{c}=1.473 \mathrm{~mm}$ ). All other conditions are identical to the base case as specified in Table 1. The front and back walls were covered with thick insulating material in order to eliminate heat losses from these surfaces $\left(\dot{q}_{f b}=0\right)$.

The effective component height was selected using Case 7 with the highest power level. The selection process consisted of matching the average board temperature rise from the simulation with the experimental value. Through a trial and error process, a component height of $h_{c}=1.473 \mathrm{~mm}$ (0.058 in.) was found to give a good match between the measured and predicted average board temperature rises (see Table 3, Case 7).

With the component height for the model selected through the matching of a single average temperature, Fig. 5 shows a comparison of measured and predicted local board temperatures for Case 7 for PCBs 1, 4, and 6. Overall, the model does predict the temperature distributions in (i) the $y$-direction including the decrease of the temperatures near the top of the PCBs due to radiation losses (see Section 4.4) and (ii) the $x$ direction, where PCBs 4 and 6 have higher temperatures than

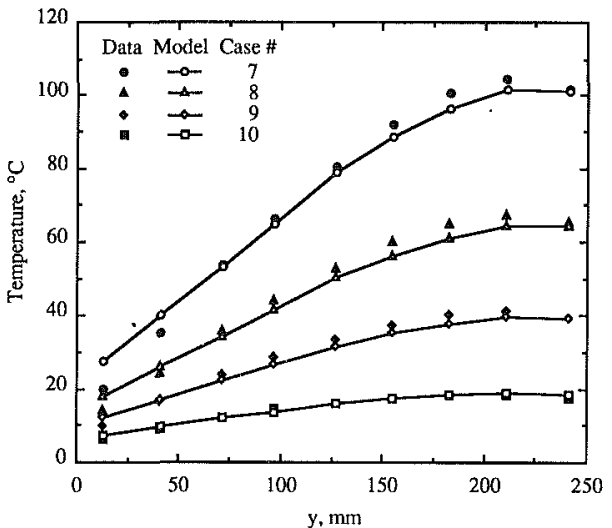

Fig. 5 Comparison of measured and predicted local board tempera tures for Case 7

PCB 1 due to heat losses from the side walls. Near the inlet, the model results are higher than the experimental data with the maximum difference being about $8 \mathrm{~K}$ for PCB 4. There is good agreement over the center portion of the PCBs. Near the outlet, the model results are slightly higher than the data with a maximum difference of $0.5 \mathrm{~K}$ for PCBs 1 and 6 . The relatively large differences near the inlet are probably due to uncertainties in the modeling of the inlet conditions. The differences could be caused by temperature variations over the depth of the PCB in the experiments (although they were found to be relatively small at mid-height, see Fig. 2), uncertainties in the temperature of the surroundings, an inaccurate knowledge of some of the thermophysical properties of the various solid materials (in particular the PCBs), and the overall heat transfer coefficients at the vertical side walls of the module (which can be expected to vary with height).

The model was then executed, with the component height unchanged from the previously selected value, for the other three power levels (Cases 8, 9, and 10). Table 3 shows that the predicted average board temperature rise is in excellent agreement with the measured value for all power levels. This indicates that for a given geometry, the effective component height for the model needs to be selected only once and is valid over a wide range of power levels and, hence, air flow rates. Similar findings were made by Ortega and Moffat $(1985,1986)$ and Moffat and Ortega (1986) who proposed in their analysis to obtain the drag properties from forced flow measurements at known flow rates. A comparison of measured and predicted local board temperatures for Cases 7 to 10 is presented in Fig. 6 . The agreement for all power levels is similar as observed for Fig. 5.

5.2 Depth Heat Loss Rate. In order to investigate the effects of heat losses from losses from the front and back walls of an electronic module and their representation in the present two-dimensional model, additional experiments at various power levels were conducted with the insulation at the front and back walls removed. The measured average board temperature rises are shown in Table $4(a)$. With the insulation removed (Cases 11 to 14 ), the average board temperature rises are between 6 to 14 percent lower than the ones with insulation (see Table 3, Cases 7 to 10 ). This represents a significant decrease and makes the inclusion of front and back heat losses in the model desirable.

In the model, the front and back heat losses are represented as a uniform heat loss rate over the entire two-dimensional domain, $\dot{q}_{f b}$ (see Eq. (4)). In order to determine numerically this heat loss rate, the model was executed for each case in Table 4 by varying the value of $\dot{q}_{f b}$ until the predicted average board temperature rise agreed with the experimental value. The previously determined component height of $h_{c}=1.473$ 


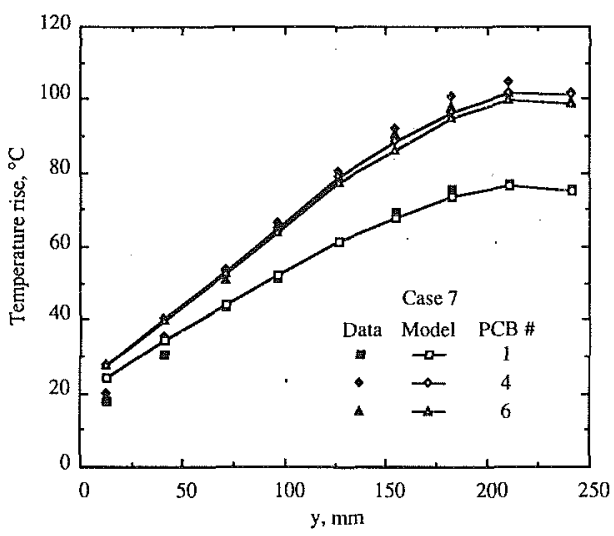

Fig. 6 Comparison of measured and predicted temperatures of PCB4 for different power levels

$\mathrm{mm}$ was used in the simulations. The resulting heat losses are given in Table 4 as heat loss rates per unit depth of the module. For the highest power level (Case 14), the depth heat loss rate constitutes about 8 percent of the total power input to the module. As expected, the heat loss rate decreases with decreasing power level and, hence, module wall temperatures. Also shown in Table $4(a)$ are the measured and predicted mean outlet velocities. Although not matched, they agree well for all power levels.

Considerable variability exists in the numerically determined heat loss rates for experiments with nearly identical conditions conducted at different times, which is illustrated in Table $4(b)$. Although the average board temperature rises show a range of less than $1.6 \mathrm{~K}$ for the highest power level (Case 14), the front and back heat loss rates vary by about 15 percent from the mean of the three tests. Obviously, experimental uncertainties could be responsible for this variation.

The heat loss from the front and back walls is estimated from the following equation

$$
q_{f b}=U\left(A_{b}+A_{f}\right)\left(T_{w}-T_{a}\right)
$$

where $U$ is the overall heat transfer coefficient for the walls, $A_{b}$ and $A_{f}$ are the back and front wall areas, and $T_{w}$ is the average temperature of the front and back walls. The value for $U$ is taken to be the same as for the side walls (see Table 1) and $A_{f}=A_{b}=2.077 \times 10^{-2} \mathrm{~m}^{2}$ (32.193 in. ${ }^{2}$ ). From an examination of the predicted side wall temperatures, the average front/back temperature rise, $\left(T_{w}-T_{a}\right)$, for Case 14 (highest power level) was estimated to be approximately $20 \mathrm{~K}$. This yields a heat loss of $q_{f b}=8.5 \mathrm{~W}$, which can be converted to a heat loss rate per unit depth of the module of $67 \mathrm{~W} / \mathrm{m}$ (Beckermann and Smith, 1992). This value agrees well with the heat loss rate found in Table 4 for Case 14, which indicates that the use of the simulation model for determining the heat loss rates gives reasonable results. It also shows that the estimation of the front and back heat losses by Eq. (9) could provide valuable input for the present model and that it is possible to account for depth heat losses in a two-dimensional model.

\section{Conclusions}

A two-dimensional model has been developed of the flow and heat transfer in a multi-board electronic module. The model includes all heat transfer modes of conduction, convection and radiation, and accounts for discrete heat generating components. Numerical studies reveal the importance of utilizing fully temperature dependent air properties and good estimates of the thermal conductivities of the various solid materials, in particular the PCBs. In addition, it is shown that the neglect of multi-dimensional radiation leads not only to
Table 4 Predicted front and back heat loss rates (a) Effect of different power levels

\begin{tabular}{ccccccc}
\hline & \multicolumn{5}{c}{$\Delta$} & \multicolumn{2}{c}{$\Delta \bar{T}_{b}, \mathrm{~K}$} & & \\
Case & $10^{5} \mathrm{~W} / \mathrm{m}^{3}$ & Exp & Sim & \multicolumn{1}{c}{ Exp } & \multicolumn{1}{c}{ Sim } & $\mathrm{W} / \mathrm{m}$ \\
\hline 11 & 0.854 & 11.3 & 11.26 & 3.56 & 3.28 & 18 \\
12 & 2.563 & 25.2 & 25.23 & 7.11 & 6.30 & 18 \\
13 & 5.121 & 41.3 & 41.34 & 10.16 & 8.84 & 18 \\
14 & 9.406 & 63.5 & 63.51 & 11.68 & 11.42 & 60 \\
\hline
\end{tabular}

(b) Variability

\begin{tabular}{lccccc}
\hline & $\dot{q}$ & $T_{e}$ & \multicolumn{2}{c}{$\Delta \bar{T}_{b}, \mathrm{~K}$} & $q_{f b}^{\prime}$ \\
Case & $10^{5} \mathrm{~W} / \mathrm{m}^{3}$ & $\mathrm{~K}$ & Exp & $\operatorname{Sim}$ & $\mathrm{W} / \mathrm{m}$ \\
\hline 13 & 5.121 & 296.9 & 41.3 & 41.34 & 18 \\
13.1 & 5.127 & 296.9 & 40.3 & 40.26 & 36 \\
13.2 & 5.112 & 296.3 & 40.4 & 40.41 & 32 \\
\hline 14 & 9.406 & 297.4 & 63.5 & 63.51 & 60 \\
14.1 & 9.404 & 295.8 & 62.4 & 62.41 & 72 \\
1.42 & 9.383 & 295.7 & 61.9 & 61.86 & 80 \\
\hline
\end{tabular}

inaccurate predictions of the temperature levels, but also to qualitatively different trends in the temperature distribution.

The model is compared to experiments conducted using a specially instrumented test setup consisting of eight vertically oriented PCBs, each of which is loaded with forty resistors, contained in an aluminum chassis that is open at the top and bottom. It is found that the model should be calibrated by selecting an effective component height that gives an accurate two-dimensional representation of the drag properties of the actual three-dimensional component/channel geometry. This was accomplished by matching of the measured and predicted average board temperature rise in a single experiment. The calibrated model gave good agreement with measured air velocities and local board temperatures over a wide range of power levels. Heat losses in the depth direction can be accurately represented in the two-dimensional model by using estimates of their magnitude.

It is concluded that the two-dimensional model allows for a prediction of the thermal behavior of complex electronic modules with some experimental calibration. This calibration is particularly needed for the present case where discrete components extend over a significant portion of the space between the PCBs. Experimental determination of the drag properties can be expected to become less important for a decreasing component height to channel width ratio (Bar-Cohen, 1985). Nevertheless, the present two-dimensional model offers information on the precise temperature profiles in the module, which can generally not be obtained from empirical or analytical thermal design methods. Considering the relatively fine numerical grid that is necessary to represent the geometry of a typical module, fully three-dimensional simulations are presently outside the capabilities of even modern computer workstations, despite the fact that 3-D flow and heat transfer (with simplified radiation) codes are commercially available. Nonetheless, the present study represents a first step in this direction and clarifies a number of issues that are equally important in more advanced models.

\section{Acknowledgments}

The authors wish to acknowledge support for this study by Rockwell International Corporation. One of the authors (C. B.) was partially supported by the National Science Foundation under grant CTS-8957149.

\section{References}

Aung, W., Beitin, K. I., and Kessier, T. J., 1972, "Natural Convection Cooling of Electronic Cabinets Containing Arrays of Vertical Circuit Cards," ASME Paper 72-WA/HT-40. 
Bar-Cohen, A., 1985, "Bounding Relations for Natural Convection Heat Transfer From Vertical Printed Circuit Boards," Proc. of the IEEE, Vol. 73, pp. 1388-1395.

Beckermann, C., and Smith, T. F., 1991, "Numerical Analysis of Combined Mode, Two-Dimensional Heat Transfer in Electronic Enclosure," Technical Report ME-TFS-91-003, Department of Mechanical Engineering, The University of Iowa, Iowa City, lowa

Beckermann, C., and Smith, T. F., 1992, "Development of a ComputerAided Simulation Model for Thermal Design of Electronic Packages," Technical Report ME-TFS-92-005, Department of Mechanical Engineering, The University of lowa, Iowa City, Iowa.

Beckermann, C., and Smith, T. F., 1993, "Incorporation of Internal Surface Radiant Exchange in the Finite Volume Method," Numerical Heat Transfer, Vol. 23, Part B, pp. 127-133.

Birnbreier, H., 1981, "Experimental Investigations on the Temperature Rise of Printed Circuit Boards in Open Cabinets With Natural Ventilation," Heat Transfer in Electronic Equipment, HTD-Vol. 20, ASME, New York, pp. 1923.

Coyne, J. C., 1984, "An Analysis of Circuit Board Temperatures in Electronic Equipment Frames Cooled by Natural Convection," Fundamentals of Natural Convection/Electronic Equipment Cooling, HTD-Vol. 32, ASME, New York, pp. 59-65.

Hawkins, L. E., and Nelson, D. J., 1992, "A Design Methodology for Vertical Channel Flow and Heat Transfer," Proc, of 1992 IEEE Inter Society Conference on Thermal Phenomena, pp. 15-22.

Incropera, F. P., and DeWitt, D. P., 1990, Fundamentals of Heat and Mass
Transfer, 3rd ed., John Wiley \& Sons, New York.

Moffat, R. J., and Ortega, A., 1986, "Buoyancy Induced Forced Convection," Heat Transfer in Electronic Equipment-1986, HTD-Vol. 57, ASME, New York, pp. 135-144.

Patankar, S. V., 1980, Numerical Heat Transfer and Fluid Flow, Hemisphere Publishing, New York.

Peterson, G. P., and Ortega, A., 1990, "Thermal Control of Electronic Equipment and Devices," Advances in Heat Transfer, J. P. Hartnett and T. F. Irvine, Jr., eds., Vol. 20, Academic Press, San Diego, pp. 181-300.

Ortega, A., and Moffat, R. J., 1986, "Buoyancy Induced Convection in Non-Uniformly Heated Array of Cubical Elements on a Vertical Wall," Heat Transfer in Electronif Equipment-1986, HTD-Vol. 57, ASME, New York, pp. 123-134.

Ortega, A., and Moffat, R. J., 1985, "Heat Transfer From an Array of Simulated Electronic Components: Experimental Results for Free Convection With and Without a Shrouding Wall," Heat Transfer in Electronic EquipmentI985, HDT-Vol. 48, ASME, New York, pp. 5-15.

Sánchez, A., and Smith, T. F., 1992, "Surface Radiant Exchange for TwoDimensional Rectangular Enclosures Using the Discrete-Ordinates Methods," ASME Journal of Heat Transfer, Vol. 114, pp. 465-472.

Smith, T. F., Beckermann, C., and Weber, S. W., 1991, "Combined Conduction, Natural Convection, and Radiation Heat Transfer in an Electronic Chassis," ASME Journat, of Electronic Packaging, Vol. 113, pp. 382-391,

Thornton, D. M., 1987, Thermal Evaluation Report: Passive Cooling Study, $L R U$ Design, Avionics Thermal Laboratory Document No. 5438AV, Rockwell International Corporation, Cedar Rapids, Iowa. 